\title{
Changes in human mesenchymal stem cell behaviors on dendrimer-immobilized surfaces due to mediation of fibronectin adsorption and assembly
}

\author{
Yuuki Ogawa, Mee-Hae Kim, and Masahiro Kino-oka* \\ Department of Biotechnology, Graduate School of Engineering, Osaka University, 2-1 Yamadaoka, \\ Suita, Osaka 565-0871, Japan \\ *Corresponding author. \\ E-mail: kino-oka@bio.eng.osaka-u.ac.jp \\ Tel: +81-(0)6-6879-7444 \\ Fax: +81-(0)6-6879-4246
}

Running title: CHANGES IN CELL BEHAVIORS BY MEDIATION OF FIBRONECTIN

Foornote: Much of this work forms the basis of the Ph.D. dissertation of Yuuki Ogawa. 


\section{Abstract}

Dynamic changes of morphologies in human mesenchymal stem cells (hMSCs) were

3 investigated on dendrimer surfaces with different capacities for fibronectin adsorption by changing

4 the polymeric generation numbers of first (G1), third (G3), and fifth (G5) generations. The amount

5 of adsorbed fibronectin on dendrimer surfaces increased with the generation number. Time-lapse

6 observations revealed that cells on the G1 surface maintained their shape with formation of

7 fibronectin fibrils in the bodies, introducing to the stabilization of focal adhesion with enriched

8 stress fibers. Cells on the G3 surface showed partial contraction with degradation of fibril structures

9 in the trailing edge. Cells on the G5 surface changed the shape by active extension and strong

10 contracting without stabilization of focal adhesion through the formation of fibronectin aggregates

11 and immature stress fibers. In addition, the paxillin which is a focal adhesion protein at lamellipodia

12 was phosphorylated, leading to active lamellipodium protrusions. These results indicate that the

13 amount and structure of fibronectin affects dynamic hMSC behaviors through the formation of

14 cytoskeletons and focal adhesions.

15

16 [Key words: dendrimer-immobilized surface, human mesenchymal stem cells, cell behaviors, 17 fibronectin adsorption on surface, fibronectin assembly] 


\section{INTRODUCTION}

2 In the culture of anchorage-dependent cells, designing the culture surface is one of the most

3 important strategies to regulate cellular functions such as migration, growth, and differentiation $(1,2)$.

4 The adhesion of cells onto a culture surface involves interactions between integrins of the cells and

5 the extracellular matrix (ECM) adsorbed on the surface (3). Fibronectin is an abundant and

6 ubiquitous ECM component that supports cell adhesion by displaying integrin-binding domains (4).

7 On a fibronectin-coated surface, morphological variation of the cells occurs based on the density of

8 binding sites which can be regulated by the coating concentration of fibronectin (5). As an alternative

9 method, Keselowsky et al. (6) reported that the charge of the culture surface by displaying functional

10 groups modulates the amount of fibronectin adsorption and its orientation to display integrin-binding

11 domains. Surface chemistry can also be manipulated to affect cellular functions through regulation of

12 fibronectin adsorption and assembly (6-8). Rico et al. (8) reported that the formation of fibronectin

13 fibrils is influenced by surface chemistry, leading to morphological changes of the cells.

14 Interactions between integrins and fibronectin on the surface form focal contact, leading to the 15 polymerization of fibronectin through the conformation changes $(4,9,10)$. Fibronectin polymerization 16 promotes stabilization of cell-matrix adhesions that are responsible for the morphological changes of 17 cells with F-actin fiber formation $(11,12)$. Therefore, morphological changes are considered to be due 18 to both the density and stability of cell-substrate adhesions.

19 In previous studies, culture surfaces that immobilize polyamidoamine dendrimers have been 20 proposed as a tool to regulate cell morphologies by modification of surface roughness, electrostatic 21 charge, and ligand density, depending on the generation number (13-16). In addition, morphological

22 variations of human mesenchymal stem cells (hMSCs) on dendrimer surfaces with different 23 generation numbers induce directed differentiation of hMSCs toward a muscle cell fate commitment $24(15,16)$. In the present study, we investigated the possible mechanisms of dynamic cell behaviors in 25 terms of focal adhesion and cytoskeletal formations of hMSCs on dendrimer surfaces with various 
generation numbers by considering the mediation of fibronectin adsorption and assembly.

\section{MATERIALS AND METHODS}

Cells and culture conditions Bone marrow-derived hMSCs (Lot no. 0000183402; Lonza,

4 Walkersville, MD, USA) were grown on tissue culture-treated polystyrene (PS) surfaces (Corning

5 Costar, Cambridge, MA, USA) in MSC growth medium (Lonza) at $37^{\circ} \mathrm{C}$ in a humidified atmosphere

6 containing $5 \% \mathrm{CO}_{2}$ as routine subcultures. At $70 \%$ confluence on the culture surface, the cells were

7 passaged by enzymatic treatment with a $0.1 \%$ trypsin $/ 0.02 \%$ EDTA solution (Sigma-Aldrich, St.

8 Louis, MO, USA). Cells that underwent less than five passages were used in experiments.

9 For all experiments, hMSCs were expanded for the indicated number of days in Dulbecco's modified

10 Eagle's medium (DMEM; Sigma-Aldrich) supplemented with 10\% fetal bovine serum (FBS) and

11 antibiotics (Life Technologies, Carlsbad, CA, USA) at a depth of $4 \mathrm{~mm}$ in the culture vessels. The

12 seeding density was $5.0 \times 10^{3}$ cells $/ \mathrm{cm}^{2}$, and medium changes were performed every 3 days.

13 Preparation of dendrimer-immobilized surfaces The PS surface of a square 8-well plate 14 (Nunc, Roskilde, Denmark) and $35 \mathrm{~mm}$ culture dish (Ibidi GmbH, Martinsried, Germany) were used 15 as starting materials as described previously (16). In brief, the hydroxyl groups were displayed on the 16 starting material by addition of potassium tert-butoxide. Next, dendron structures were established

17 by reactions of glutaraldehyde and tris (2-aminoethyl) amine solutions, which were repeated for the

18 suitable generation numbers. A D-glucose solution was kept in the vessels and a sodium borohydride

19 solution was added to the vessels without removal of the D-glucose solution. Subsequently, the dishes

20 were washed with sterile water before use of the dendrimer-immobilized surfaces.

21 Western blotting Protein collection and western blotting were carried out as described in 22 previous report (13). After incubation with culture medium, the culture surfaces were rinsed with 23 phosphate-buffered saline (PBS; Sigma-Aldrich) twice and adsorbed protein was dissolved in lysis 24 buffer (CelLytic M; Sigma-Aldrich) for $15 \mathrm{~min}$ at room temperature. After harvesting the protein 25 samples by scraping and centrifugation at $4{ }^{\circ} \mathrm{C}$, the supernatant was collected. The incubated medium 
1 was reacted with $20 \%$ trichloroacetic acid for $30 \mathrm{~min}$ at $4^{\circ} \mathrm{C}$. After centrifugation, the precipitates

2 were washed with acetone twice and then dissolved in the lysis buffer for $15 \mathrm{~min}$ at room

3 temperature. After centrifugation at $4^{\circ} \mathrm{C}$, the supernatant was collected. The protein samples were

4 mixed with Laemmli buffer and then heated at $95^{\circ} \mathrm{C}$ for $10 \mathrm{~min}$. Proteins were separated by

5 SDS-polyacrylamide gel electrophoresis and transferred to polyvinylidene difluoride nylon

6 membranes (Hybond-P; GE Healthcare, Buckinghamshire, UK). After masking the non-specific

7 proteins (ECL Blocking Agent; GE Healthcare) at room temperature for $2 \mathrm{~h}$, the membrane was

8 reacted with a primary antibody against fibronectin (Santa Cruz Biotechnology, Dallas, TX, USA) at

$9 \quad 4^{\circ} \mathrm{C}$ overnight. The membrane was washed with PBS containing $0.1 \%$ Polyoxyethylene (20) sorbitan

10 monolaurate (PBS-T), followed by the incubation with a peroxidase-linked secondary antibody (GE

11 Healthcare) at room temperature for $1 \mathrm{~h}$. The membranes were washed with PBS-T and reacted with

12 detection reagent (ECL Detection Reagent; GE Healthcare) for 1 min before exposure to X-ray film

13 (Fujifilm, Tokyo, Japan).

14 Immunofluorescence staining Cells were fixed with 4\% paraformaldehyde in PBS for 10

$15 \mathrm{~min}$ at room temperature and then rinsed with PBS twice. The cells were then incubated in PBS with

$16 \quad 0.5 \%$ polyoxyethylene (10) octylphenyl ether for $10 \mathrm{~min}$. After masking the non-specific proteins

17 (Block Ace; Dainippon Sumitomo Pharma Co., Ltd., Osaka, Japan) for $90 \mathrm{~min}$ at room temperature,

18 the cells were incubated with primary antibodies against fibronectin, paxillin (Millipore, Billerica,

19 MA, USA), and phosphorylated-paxillin (Cell Signaling Technology Inc., Danvers, MA, USA) at

$204{ }^{\circ} \mathrm{C}$ overnight. The cells were washed with Tris-buffered saline (TBS; Dako, Glostrup, Denmark)

21 and reacted with secondary antibodies (Alexa Flour 488-conjugated anti-mouse IgG or Alexa Flour

22 594-conjugated anti-rabbit IgG; Life Technologies) for $1 \mathrm{~h}$ at room temperature. Nuclei and F-actin

23 were stained with 4',6-diamidino-2-phenylindole and fluorescence-phalloidin (Life Technologies),

24 respectively. Images were obtained using a confocal laser scanning microscope (FV-1000; Olympus,

25 Tokyo, Japan) through a $60 \times$ objective lens. 
Time-lapse fluorescence observations The cells were transfected with actin-RFP by

2 baculovirus (CelLight actin-RFP; Life Technologies) according to the commercially available

3 protocol. Then, the cells were cultured in DMEM with $10 \%$ FBS containing $5 \mu \mathrm{g} / \mathrm{ml}$

4 fluorescence-labeled fibronectin (Cytoskeleton, Denver, CO, USA). Images were obtained every 5

5 min using a time-lapse confocal laser scanning microscope (FV-10i; Olympus) through a $60 \times$

6 objective lens.

7 Quantitative RT-PCR RNA isolation and quantitative RT-PCR assays were carried out as

8 described previously (13). Total RNA was extracted from the cells (RNeasy Mini Kit; Qiagen, Hilden,

9 Germany) according to the commercially available protocol. Reverse transcription was conducted

10 (SuperScript II Reverse Transcriptase kit; Takara Bio Inc., Shiga, Japan). Quantitative PCR assays

11 were carried out (SYBR Premix EX Taq; Takara Bio Inc. and 7300 Real-Time PCR System; Applied

12 Biosystems, Foster City, CA, USA). Specific primers were membrane type 1-matrix

13 metalloproteinase (MT1-MMP), forward 5'-CCATTGGGCATCCAGAAGAGAGC-3'; reverse

14 5'-GGATACCCAATGCCCATTGGCCA-3' and glyceraldehyde-3-phosphate dehydrogenase

15 (GAPDH), forward 5'-CAACGGATTTGGTCGTATTGG-3'; $\quad$ reverse

16 5'-GCCATGGGTGGAATCATATTG-3' according to previous reports $(13,17)$. The relative

17 quantitation of target mRNA expression was estimated by the cycle threshold value $\left(C_{\mathrm{t}}\right)$. Relative 18 mRNA expression was normalized to the GAPDH expression to obtain the $\Delta C_{\mathrm{t}}$ value and calculated 19 by $2^{-\Delta C t}$

\section{RESULTS}

21 Adsorption of fibronectin on dendrimer surfaces Western blotting was carried out using

22 samples of the proteins adsorbed on G1, G3, and G5 surfaces and the unadsorbed proteins in the

23 supernatant after 1 day of incubation with 10\% FBS/DMEM. As shown in Fig. 1, the amount of Fig. 1

24 adsorbed fibronectin on the surfaces increased with the generation number of the dendrimer surface,

25 while a decrease in the amount of fibronectin occurred in the medium. 
Cytoskeletal formation and fibronectin assembly Cytoskeletal formation and fibronectin assembly were observed by immunostaining of F-actin and fibronectin in hMSCs on the G1, G3, and G5 surfaces at days 1 and 3. As shown in Fig. 2, the spindle-shaped cells on all surfaces Fig. 2 showed abundant F-actin filaments at day 1. Fibronectin was detected on all surfaces but was concentrated in the interfaces between cells and surfaces (Fig. S1). Fibril and small structures of fibronectin were found on the surfaces (Fig. 2), indicating fibronectin fibrils $\left(10^{1}-10^{3} \mu \mathrm{m}\right.$ in length) and fibronectin aggregates $\left(10^{0}-10^{1} \mu \mathrm{m}\right.$ in diameter), respectively (18). In particular, fibril structures were not detected in the cell edge (Fig. 2A1, B1, and C1), and similarly to the formation

9 of stress fibers, much more fibril structures were observed of the longitudinal direction in cell 10 bodies on the G1 and G3 surfaces, compared with those on the G5 surface (Fig. 2A-C). At day 3, 11 on all surfaces, little fibronectin fibril was detected in the cell edge. In addition, the spindle-shaped 12 cells on the G1 surface maintained the formation of stress fibers, and the fibronectin fibrillogenesis 13 was promoted in the bodies, compared with those at day 1 (Fig. 2D). On the G3 surface, stress 14 fibers and fibril structures were localized in half side of the bodies along the major axis (Fig. 2E2), 15 although little fibronectin fibril was detected in the other one (see the arrow in Fig. 2E). In contrast, 16 on the G5 surface, both the round- and spindle-shaped cells showed many lamellipodia with less 17 stress fibers, compared with those on the other surfaces. And the fibronectin formed small compact 18 structures in the cell bodies (Fig. 2F and G).

Actin-RFP expressing hMSCs were observed with fluorescent labeled-fibronectin on the G1,

20 G3, and G5 surfaces at day 3. On the G1 surface, spindle-shaped cells showed lamellipodia with

21 little fibril structures in the leading edge and stress fibers with fibrill structures in the bodies. With 22 extension of the cells, fibronectin fibrillogenesis was seen in the bodies along the direction of 23 migration. Then, the cells contracted their trailing edge with shortening of stress fibers and 24 fibronectin fibrils (Movie S1). On the G3 surface, spindle-shaped cells showed lamellipodium 25 formation with little fibronectin fibril in the leading and trailing edges as well as stress fibers with 
1 fibronectin fibrils in the bodies. Upon extension of the cells, it was observed that lamellipodium

2 protrusions with fibronectin fibrillogenesis in the front-side of the bodies and partial contraction of

3 the cells through release of trailing edge with disruption of fibronectin fibrils (Movie S2 and Fig.

4 S1). Furthermore, on the G5 surface, round-shaped cells showed lamellipodia with little fibril

5 structures in whole bodies. The cells extended the shape to bipolar directions with active

6 lamellipodium protrusions. Upon the extension of the cells, immature stress fibers and small

7 structures of fibronectin were formed in whole bodies. Then, the cells contracted with sudden

8 release of the trailing edge. This temporal stretching was repeated with increasing the amount of

9 small structures of fibronectin on the cells (Movie S3 and Fig. S2).

10 Enzymatic activity for matrix degradation The mRNA expression of MT1-MMP, which is

11 one of the enzymes for fibronectin degradation, was examined in hMSCs cultured on G1, G3, and Fig. 3

12 G5 surfaces at days 1 and 3. As shown in Fig. 3, relative mRNA expression of MT1-MMP in

13 hMSCs on the G5 surface was significantly higher than that on G1 and G3 surfaces at day 1 . In

14 addition, at day 3, the expression of MT1-MMP on the G1 surface was lower than that on the G3

15 surface, while the expression in hMSCs on the G5 surface at day 3 was 2-fold higher than that at 16 day 1.

17 Formation and phosphorylation of paxillin Immunostainings of F-actin, paxillin, and 18 phosphorylated-paxillin were conducted in hMSCs cultured on the G1, G3, and G5 surfaces at day

19 3. As shown in Fig. 4, paxillin spots were distributed at terminal of stress fibers at edge and bodies

20 of the cells on the G1 surface (Fig. 4A). With increasing generation numbers of the dendrimer

21 surface, the distribution of paxillin spots was more intensive at cell edge, while being less in the

22 bodies, compared to those on the G1 surface. In particular, on the G5 surface, almost all paxillin

23 had localized in the lamellipodia in the edge in both round- and spindle-shaped cells (Fig. 4C and

24 D). Furthermore, as shown in Fig. 5A-C, expression of phosphorylated-paxillin was not observed Fig. 5

25 in hMSCs cultured on G1 or G3 surfaces, although phosphorylated-paxillin spots were detected in 
1 the edge of cells on the G5 surface.

2

3

4

13 fibrillogenesis stimulates cell contraction associated with shortening of stress fibers through a

14 Rho-dependent mechanism (20-22). In our previous report (16), cells on the G1 surface exhibited the

15 higher activity of RhoA than those on the G3 and G5 surfaces. These evidences suggested that

16 fibronectin fibrillogenesis stimulates the RhoA activity to facilitate the stress fiber formation, causing

17 the migration with spindle-shape. On the G3 surface, the dynamic cell behavior (Movie S2) exhibited

18 that the cells made the extension in the leading edge, low stabilization of focal adhesion, and

19 partially strong contraction with promotion of fibronectin fibrillogenesis and stress fiber formation

20 (Fig. 6Ad and Ae), as well as with degradation of fibril structures in the trailing edge and stress fiber 21 disruption (Fig. 6Ae and Af). On the G5 surface (Movie S3), the cells changed the shape by active

The present study describes dynamic changes in behaviors of hMSCs on dendrimer surfaces with various generation numbers due to the mediation of fibronectin adsorption and assembly. These behaviors consist of the formation of attachments between integrins of extended membranes and adsorbed fibronectin on the surface, stabilization of adhesion in the cell body, and contraction of the cell rear with release of attachments from the substrate (19). Time-lapse observation (Movie S1) revealed that cells on the G1 surface migrated with maintenance of their spindle-shape through extension in the leading edge, stabilization of focal adhesion, and contraction in the trailing edge with promotion of fibrillogenesis and stress fiber formation in the bodies (Fig. 6Aa and $\mathrm{Ab}$ ), as well as with fibronectin aggregation and release of focal adhesion in the trailing edge (Fig. 6Ab and Ac), similarly to those on the conventional PS surface. It has been documented that fibronectin 
1 extension because of the adsorption of fibronectin from the surrounding components in the medium.

2 Thus, the changes in fibronectin assembly and cytoskeletal formation were responsible for the

3 dynamic cell behaviors on the dendrimer surfaces.

4 The possible processes for fibronectin adsorption and assembly associated with the formation of

5 focal adhesions and actin cytoskeletons at cell-surface interfaces were considered as follow (Fig. 6B).

6 The adsorbed fibronectin with a compact conformation is unfolded to display the $\mathrm{N}$-terminal

7 fibronectin-fibronectin binding domain, leading to the formation of fibronectin fibrils (4). It has been

8 documented that fibronectin fibrillogenesis induces the formation of fibrillar adhesion, which is

9 essential for stress fiber formation through constructing focal adhesions $(11,21)$. In the present study,

10 immunostainings indicated that cells on the G1 surface had stable focal adhesion at the terminal of

11 stress fibers in the edge and bodies, accompanied by the formation of fibronectin fibrils (Fig. 6Ab,

$12 \mathrm{~B} 1$, and B2). In contrast, on the G5 surface, localization of focal adhesion was detected in

13 lamellipodia with little formation of stress fibers and fibronectin fibrils compared with those on the

14 G1 surface (Figs. 2 and 4). Similar phenomena of fibronectin assembly have been reported by

15 Faucheux et al. (7) in cultures of human skin fibroblasts. The inhibition of fibronectin fibrillogenesis

16 occurred on a positively charged surface by showing a larger amount of fibronectin adsorption

17 compared with that on a negatively charged surface. In this study, compared with the other surfaces,

18 the amount of adsorbed fibronectin from the culture medium was larger on the G5 surface (Fig. 1).

19 On a larger amount of adsorbed fibronectin, it has been documented that the turnover of focal

20 adhesions is promoted with active cell migration through paxillin phosphorylation $(23,24)$. Paxillin

21 phosphorylation enhances the turnover of focal adhesion with lamellipodium protrusions, whereas

22 non-phosphorylated paxillin leads to the formation of fibrillar adhesions (25). In the present study,

23 phosphorylated-paxillin was detected in cells, although less phosphorylated-paxillin was found in

24 cells on the G1 and G3 surfaces (Fig. 5). These suggested that the active extension on the G5 surface

25 occurred due to high turnover of paxillin through high adsorption of fibronectin and its promotion of 
1 paxillin phosphorylation (Fig. 6B3). Furthermore, the inhibition of fibronectin fibrillogenesis

2 occurred in cells on the G5 surface (Fig. 2), which is considered to be related to matrix degradation

3 mediated by specific enzymes such as MMPs (26-28). In particular, MT1-MMP plays a role in the

4 degradation of fibronectin fibrils to promote cell motility (28), and the presence of fibronectin made

5 up-regulation of expressions for MT1-MMP (29). These evidences supported our current results of

6 higher expression of MT1-MMP in cells on the G5 surface, which was made by higher adsorption of

7 fibronectin (Figs. 1 and 3), suggesting that the unstable adhesions of cells on the G5 surface appeared

8 through no formation of fibronectin fibrils and focal adhesions in cell bodies by degradation of

9 unfolding fibronectin (Fig. 6B4). These results suggested that a dendrimer surface with high cationic

10 properties was sufficient for fibronectin adsorption and the inhibition of fibronectin fibrillogenesis

11 through MMP activation, causing the active extension and adhesion turnover with formation of

12 immature stress fibers on the G5 surface. Cells on the G3 surface with middle level of fibronectin

13 adsorption exhibited the transitional behaviors, suggesting that the partial degradation of fibronectin

14 fibrils by MMPs caused the extension and stable adhesion with fibronectin fibrillogenesis and stress

15 fiber formation in the leading edge, and partial unstable adhesion and contraction with fibronectin

16 aggregation in the trailing edge (Fig. 6Af, B1, B2, and B4).

17 In conclusion, the present study indicates that fibronectin adsorption and assembly by the cells

18 changes dynamic cell behaviors. Moreover, we found that adsorption of fibronectin induces the

19 extension of cells, and that fibronectin assembly affected by MMPs plays important roles in the

20 stabilization of focal adhesions associated with cytoskeletal formations. These results suggest that

21 control of the cell-surface interface in fibronectin adsorption and assembly represents a versatile

22 approach to manipulate specific cellular responses to biomaterials for tissue engineering applications.

\section{ACKNOWLEDGMENTS}

24 This work was partially supported by the Japan Science and Technology Agency (JST),

25 Grants-in-Aid for Scientific Research (B) (no. 21360402) from the Ministry of Education, Culture, 
1 Sports, Science and Technology of Japan, by the MEXT project, "Creating Hybrid Organs of the 2 future" at Osaka University, and by the Japan Society for the Promotion of Science (JSPS) through 3 Japanese-German Graduate Externship. 
2 1. Bacakova, L., Filova, E., Rypacek, F., Svorcik, V., and Stary, V.: Cell adhesion on artificial materials for tissue engineering, Physiol. Res., 53, S35-S45 (2004).

4 2. Higuchi, A., Ling, Q. D., Chang, Y., Hsu, S. T., and Umezawa, A.: Physical cues of 5 biomaterials guide stem cell differentiation fate, Chem. Rev., 113, 3297-3328 (2013).

$6 \quad 3 . \quad$ Wilson, C. J., Clegg, R. E., Leavesley, D. I., and Pearcy, M. J.: Mediation of biomaterial$7 \quad$ cell interactions by adsorbed proteins: a review, Tissue Eng., 11, 1-18 (2005).

8 4. Mao, Y. and Schwarzbauer, J. E.: Fibronectin fibrillogenesis, a cell-mediated matrix

5. Dolatshahi-Pirouz, A., Jensen, T. H., Kolind, K., Bunger, C., Kassem, M., Foss, M., and Besenbacher, F.: Cell shape and spreading of stromal (mesenchymal) stem cells cultured on fibronectin coated gold and hydroxyapatite surfaces, Colloids Surf. B Biointerfaces, 84, 18-

14 6. Keselowsky, B. G., Collard, D. M., and Garcia, A. J.: Surface chemistry modulates fibronectin conformation and directs integrin binding and specificity to control cell adhesion, J. Biomed. Mater. Res. A, 66, 247-259 (2003).

7. Faucheux, N., Tzoneva, R., Nagel, M. D., and Groth, T.: The dependence of fibrillar adhesions in human fibroblasts on substratum chemistry, Biomaterials, 27, 234-245 (2006).

8. Rico, P., Rodriguez-Hernandez, J. C., Moratal, D., Altankov, G., Monleon-Pradas, M., and Salmeron-Sanchez, M.: Substrate-induced assembly of fibronectin into networks: influence of surface chemistry and effect on osteoblast adhesion, Tissue Eng. Part A, 15, 3271-3281 (2009).

9. Gardel, M. L., Schneider, I. C., Aratyn-Schaus, Y., and Waterman, C. M.: Mechanical integration of actin and adhesion dynamics in cell migration, Annu. Rev. Cell Dev. Biol., 26, $315-333$ (2010). 
10. Lawson, C. and Schlaepfer, D. D.: Integrin adhesions: Who's on first? What's on second? Connections between FAK and talin, Cell Adh. Migr., 6, 302-306 (2012).

11. Sottile, J. and Hocking, C. D.: Fibronectin polymerization regulates the composition and stability of extracellular matrix fibrils and cell-matrix adhesions, Mol. Biol. Cell, 13, 35463559 (2002).

12. Huveneers, S., Truong, H., Fassler, R., Sonnenberg, A., and Danen, E. H.: Binding of soluble fibronectin to integrin $\alpha 5 \beta 1$ - link to focal adhesion redistribution and contractile shape, J. Cell Sci., 121, 2452-2462 (2008).

13. Kim. M.-H. and Kino-oka, M.: Switching between self-renewal and lineage commitment of human induced pluripotent stem cells via cell-substrate and cell-cell interactions on a dendrimer-immobilized surface, Biomaterials, 35, 5670-5678 (2014).

14. Kim, M.-H., Kino-oka, M., Kawase, M., Yagi, K., and Taya, M.: Response of human epithelial cells to culture surfaces with varied roughnesses prepared by immobilizing dendrimers with/without D-glucose display, J. Biosci. Bioeng., 103, 192-199 (2007).

15. Kim, M.-H., Kino-oka, M., Saito, A., Sawa, Y., and Taya, M.: Myogenic induction of human mesenchymal stem cells by culture on dendrimer-immobilized surface with D-glucose display, J. Biosci. Bioeng., 109, 55-61 (2010).

16. Kim, M.-H., Kino-oka, M., Maruyama, N., Saito, A., Sawa, Y., and Taya, M.: Cardiomyogenic induction of human mesenchymal stem cells by altered Rho family GTPase expression on dendrimer-immobilized surface with D-glucose display, Biomaterials, 31, 7666-7677 (2010).

17. Gilles, C., Polette, M., Coraux, C., Tournier, J. M., Menequzzi, G., Munaut, C., Volders, L., Rousselle, P., Birembaut, P., and Foidart, J. M.: Contribution of MT1-MMP and of human laminin-5 $\gamma 2$ chain degradation to mammary epithelial cell migration, J. Cell Sci., 114, 2967-2976 (2001). 
18. Pellenc, D., Berry, H., and Gallet, O.: Adsorption-induced fibronectin aggregation and fibrillogenesis, J. Colloid Interface Sci., 298, 132-144 (2006).

19. Friedl, P. and Wolf, K.: Proteolytic interstitial cell migration: a five-step process, Cancer Metastasis Rev., 28, 129-135 (2009).

Hocking, D. C., Sottile, J., and Langenbach, K. J.: Stimulation of integrin-mediated cell contractility by fibronectin polymerization, J. Biol. Chem., 275, 10673-10682 (2000).

22. Worthylake, R. A., Lemoine, S., Watson, J. M., and Burridge, K.: RhoA is required for monocyte tail retraction during transendothelial migration, J. Cell Sci., 154, 147-160 (2001).

23. Gupton, S. L. and Waterman-Storer, C. M.: Spatiotemporal feedback between actomyosin and focal-adhesion systems optimizes rapid cell migration, Cell, 125, 13611374 (2006).

24. Li, D., Ding, J., Wang, X., Wang, C., and Wu, T.: Fibronectin promotes tyrosine phosphorylation of paxillin and cell invasiveness in the gastric cancer cell line AGS, Tumori, 95, 769-779 (2009).

25. Zaidel-Bar, R., Milo, R., Kam, Z., and Geiger, B.: A paxillin tyrosine phosphorylation switch regulates the assembly and form of cell-matrix adhesions, J. Cell Sci., 120, 137-148 (2007).

26. Shi, F. and Sottile, J.: MT1-MMP regulates the turnover and endocytosis of extracellular matrix fibronectin, J. Cell Sci., 124, 4039-4050 (2011).

27. Jiao, Y., Feng, X., Zhan, Y., Wang, R., Zheng, S., Liu, W., and Zeng, X.: Matrix metalloproteinase- 2 promotes $\alpha v \beta 3$ integrin-mediated adhesion and migration of human 
melanoma cells by cleaving fibronectin, PLoS One, 7, e41591 (2012).

2 28. Takino, T., Nagao, R., Manabe, R., Domoto, T., Sekiguchi, K., and Sato, H.: Membrane-type 1 matrix metalloproteinase regulates fibronectin assembly to promote cell motility, FEBS Lett., 585, 3378-3384 (2011).

29. Esparza, J., Viardell, C., Calvo, J., Juan, M., Vives, J., Urbano-Marquez, A., Yaque, J., and Cid, M. C.: Fibronectin upregulates gelatinase B (MMP-9) and induces coordinated expression of gelatinase A (MMP-2) and its activator MT1-MMP (MMP-14) by human T lymphocyte cell lines. A process repressed through RAS/MAP kinase signaling pathway, Blood, 94, 2754-2766 (1999). 
FIGURE LEGENDS

2 FIG. 1. Western blotting of fibronectin adsorbed on the culture surfaces and remaining in the culture medium on the G1, G3, and G5 surfaces after 1 day of incubation.

FIG. 2. Immunostainings of F-actin (red), fibronectin (green), and nuclei (blue) in hMSCs cultured on the G1 (A and D), G3 (B and E), and G5 (C, F, and G) surfaces at days 1 and 3. Panels A1-G2 show enlargements of boxed areas in panels A-G, respectively. Scale bars: $50 \mu \mathrm{m}$ $(\mathrm{A}-\mathrm{G})$ and $10 \mu \mathrm{m}(\mathrm{A} 1-\mathrm{G} 2)$.

FIG. 3. Quantitative RT-PCR analysis of MT1-MMP expression in hMSCs cultured on the G1 (dark bar), G3 (gray bar), and G5 (blank bar) surfaces at days 1 and 3. Data represent average values with the standard deviation determined from at least triplicate independent experiments. Data were analyzed using the Tukey-Kramer method. Statistical significances among the data sets were considered at $* p<0.01$ and $* * p<0.05$.

FIG. 4. Immunostainings of F-actin (red), paxillin (green), and nuclei (blue) on the G1 (A), G3 (B), and G5 (C and D) surfaces at day 3. Panels A1-D2 show enlargements of boxed areas in panels A-D, respectively. Scale bars: $50 \mu \mathrm{m}(\mathrm{A}-\mathrm{D})$ and $10 \mu \mathrm{m}(\mathrm{A} 1-\mathrm{D} 2)$.

FIG. 5. Immunostainings of paxillin (green), phosphorylated-paxillin (red), and nuclei (blue) on the G1 (A), G3 (B), and G5 (C) surfaces at day 3. Panel C1 shows an enlargement of the boxed area in panel C. Scale bars: $50 \mu \mathrm{m}(\mathrm{A}-\mathrm{C})$ and $10 \mu \mathrm{m}(\mathrm{C} 1)$.

FIG. 6. Schematic illustrations of dynamic changes in behaviors of hMSCs associated with formation of focal adhesion and cytoskeleton due to the adsorption and assembly of fibronectin (A) on the G1 surface $(\mathrm{Aa}-\mathrm{Ac})$, the G3 surface (Ad-Af), and the G5 surface (Ag-Ai), and cellsurface interfaces in adsorption and assembly of fibronectin associated with the formation of focal adhesions and actin cytoskeletons (B) for cell extension (B1), stable adhesion (B2), active extension (B3), and unstable adhesion (B4). 
Fig. 1

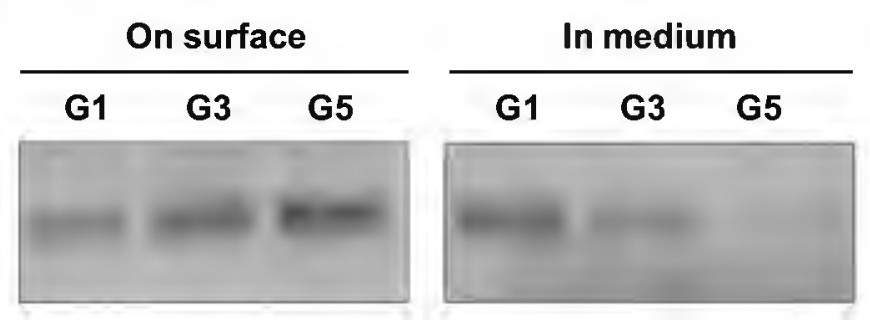


Fig. 2

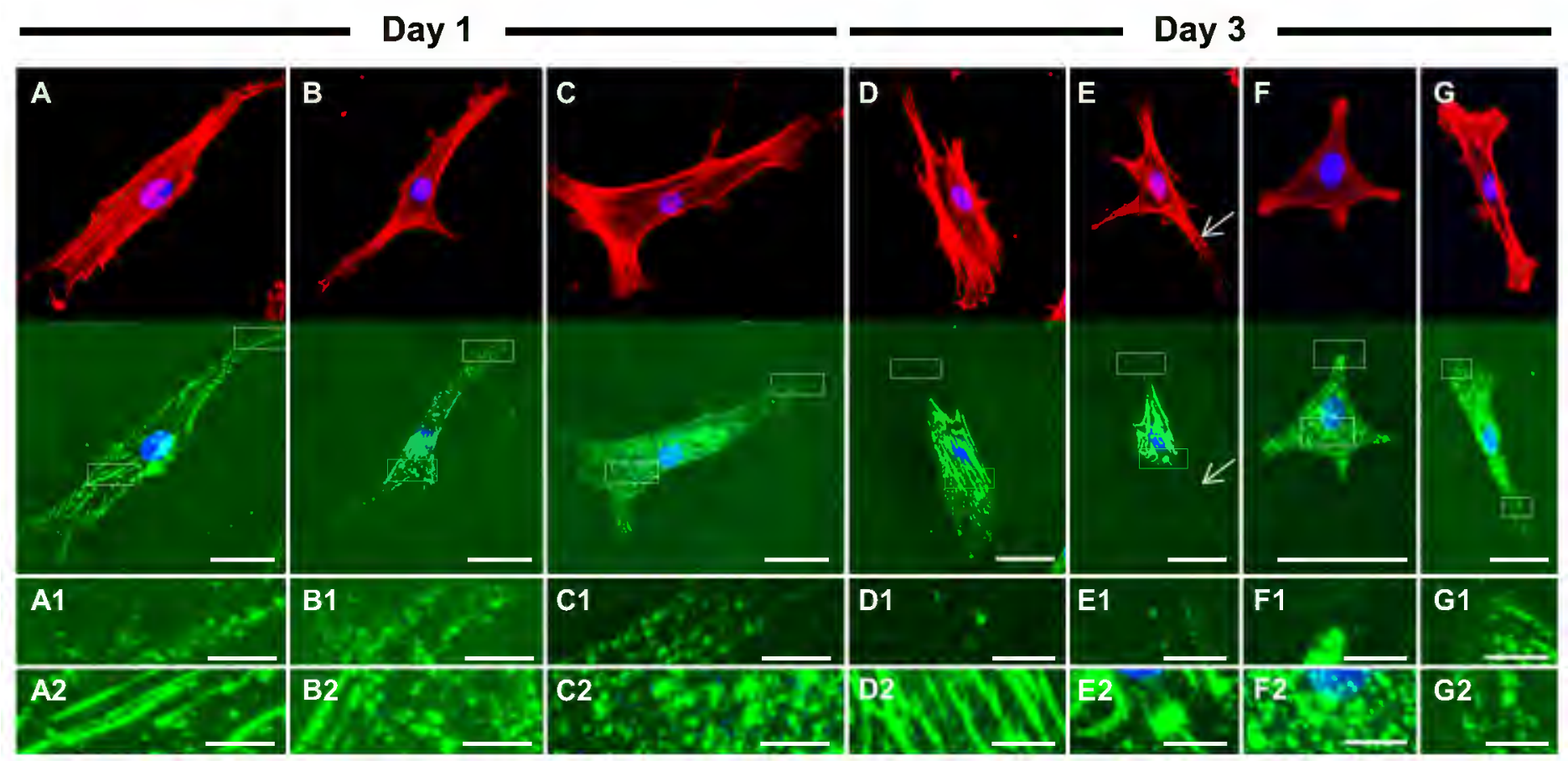


Fig. 3

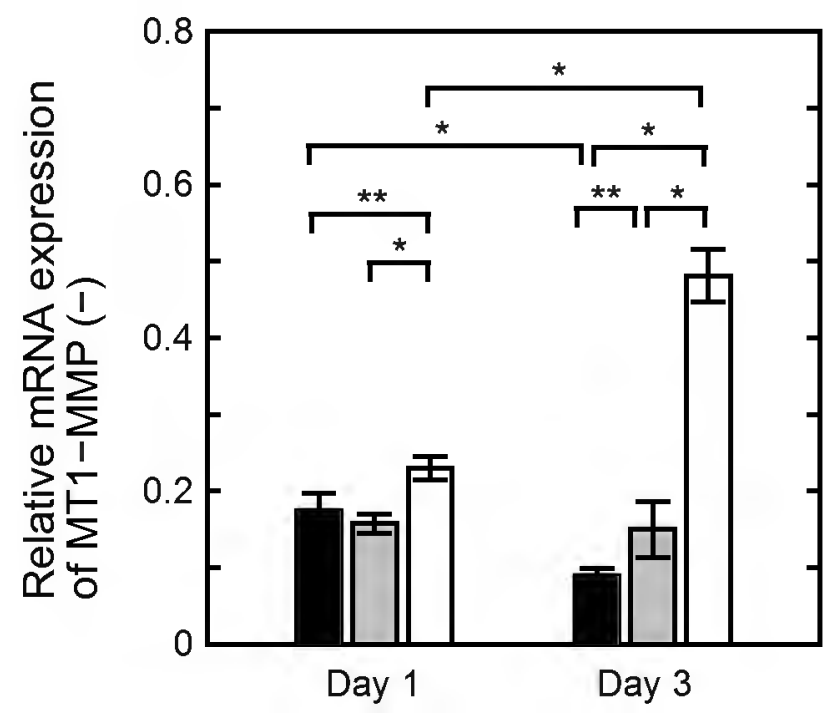


Fig. 4
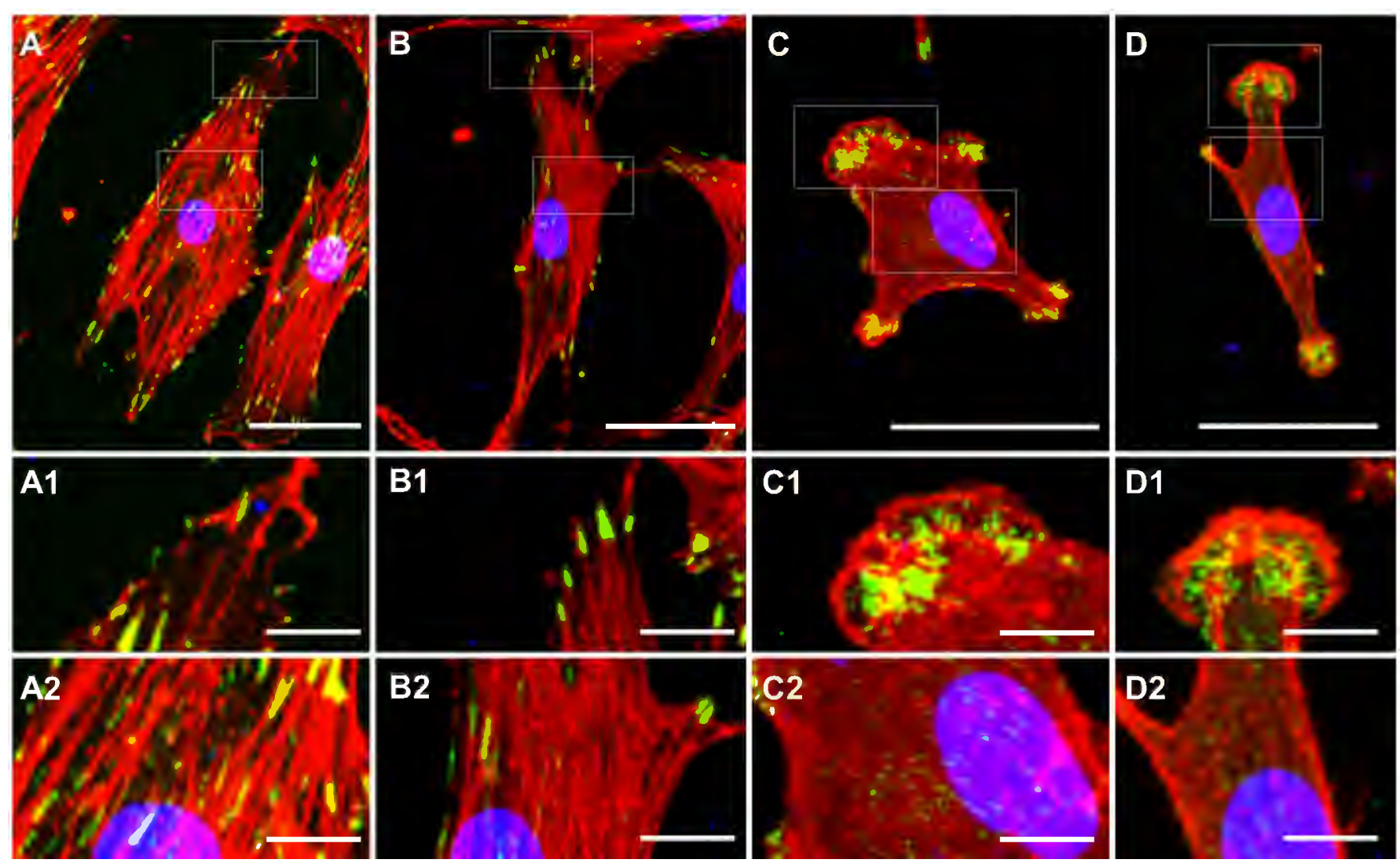
Fig. 5

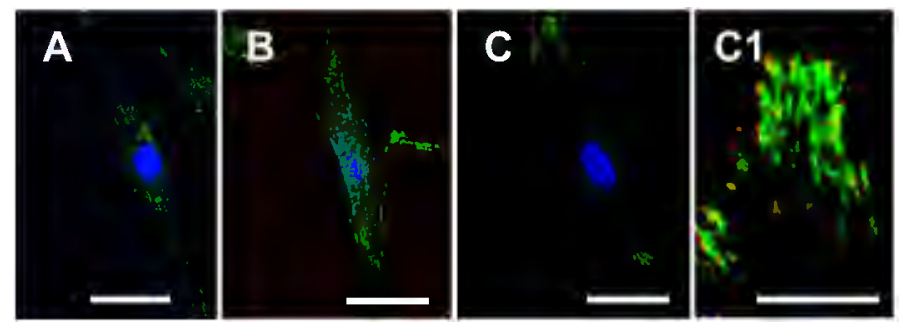


Fig. 6

A

G1 surface

G3 surface

G5 surface

Aa

Ad

extension $\leftrightarrow \propto$

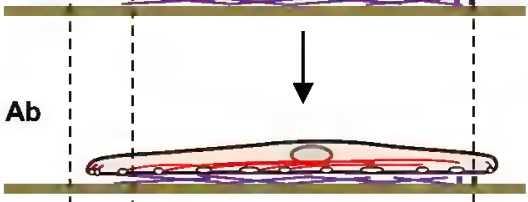

B1 B2

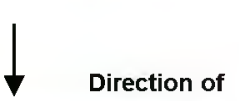

Ac contraction

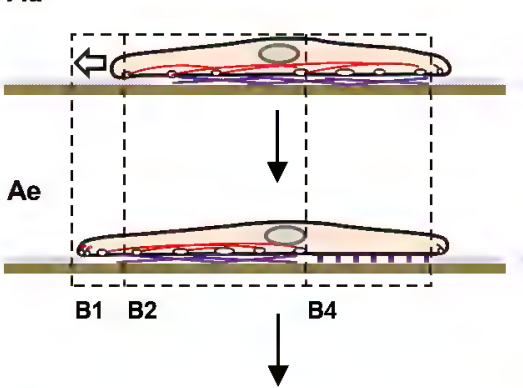

Af

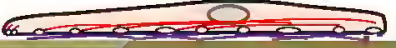
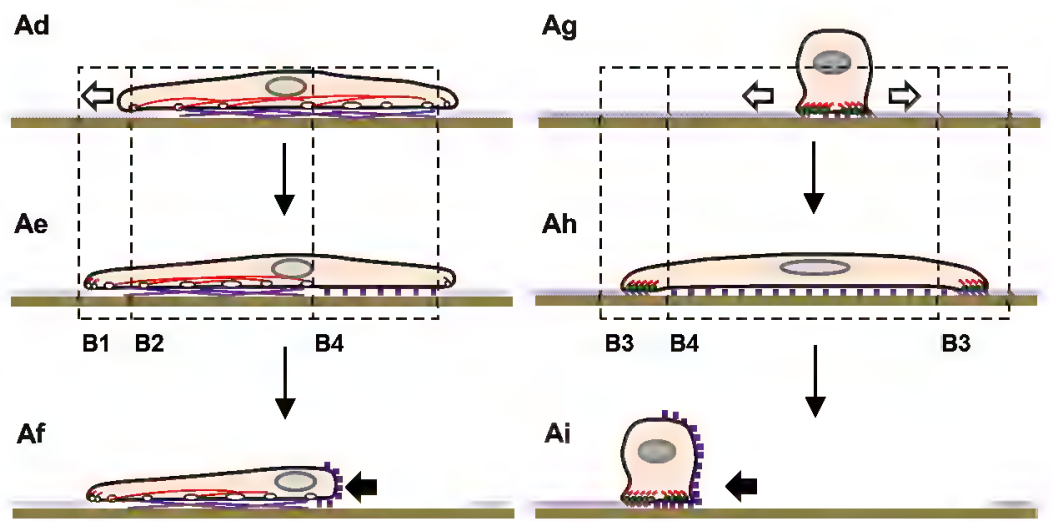

B

B1 Extension

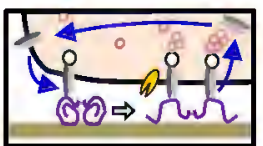

B2 Stable adhesion

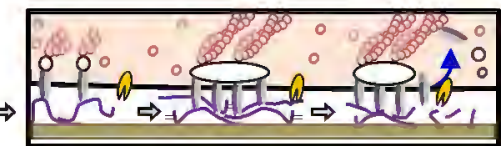

B3 Active extension

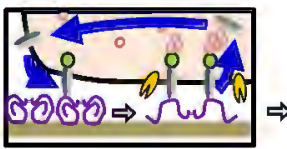

B4 Unstable adhesion

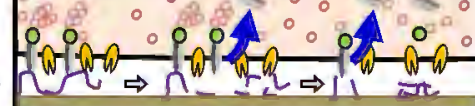

\begin{tabular}{|c|c|c|c|c|c|c|c|c|}
\hline- & Stress fiber & , & Lamellipodia & 0 & Nucleus & - Fibronectin & & \\
\hline ธ่0 & $\begin{array}{l}\text { Folding } \\
\text { fibronectin }\end{array}$ & $\Omega \Omega$ & $\begin{array}{l}\text { Unfolding } \\
\text { fibronectin }\end{array}$ & $\overline{2}$ & $\begin{array}{l}\text { Fibronectin } \\
\text { fibril }\end{array}$ & $7 \begin{array}{l}\text { Fibronectin } \\
\text { fragment }\end{array}$ & ist & $\begin{array}{l}\text { Fibronectin } \\
\text { aggregate }\end{array}$ \\
\hline$\theta$ & Integrins & 0 & Paxillin & 0 & $\begin{array}{l}\text { Phosphorylated } \\
\text { paxillin }\end{array}$ & - Actin & 0 & $\begin{array}{l}\text { Matrix } \\
\text { metalloproteinase }\end{array}$ \\
\hline
\end{tabular}

